\title{
Mechanism of the trivalent lanthanides' persistent luminescence in wide bandgap materials
}

\author{
Leipeng Li ${ }^{1}$, Tianyi Li ${ }^{1}$, Yue Hu${ }^{1}$, Chongyang Cai ${ }^{1}$, Yunqian $\mathrm{Li}^{1}$, Xuefeng Zhang ${ }^{1}$, Baolai Liang ${ }^{1,2}$, Yanmin Yang ${ }^{1 凶}$ and \\ Jianrong Qiu (1) ${ }^{3 凶}$
}

\begin{abstract}
The trivalent lanthanides have been broadly utilized as emitting centers in persistent luminescence (PersL) materials due to their wide emitting spectral range, which thus attract considerable attention over decades. However, the origin of the trivalent lanthanides' PersL is still an open question, hindering the development of excellent PersL phosphors and their broad applications. Here, the PersL of 12 kinds of the trivalent lanthanides with the exception of $\mathrm{La}^{3+}, \mathrm{Lu}^{3+}$, and $\mathrm{Pm}^{3+}$ is reported, and a mechanism of the Pers L of the trivalent lanthanides in wide bandgap hosts is proposed. According to the mechanism, the excitons in wide bandgap materials transfer their recombination energy to the trivalent lanthanides that bind the excitons, followed by the generation of PersL. During the PersL process, the trivalent lanthanides as isoelectronic traps bind excitons, and the binding ability is not only related to the inherent arrangement of the $4 \mathrm{f}$ electrons of the trivalent lanthanides, but also to the extrinsic ligand field including anion coordination and cation substitution. Our work is believed to be a guidance for designing high-performance PersL phosphors.
\end{abstract}

\section{Introduction}

Persistent luminescence (PersL) phosphors can continue to emit light for seconds and even longer after the stoppage of excitation. Due to this attractive feature, PersL phosphors have a wide range of applications in the fields of display, anti-counterfeiting, information storage, biological labeling, etc. ${ }^{1-8}$. In general, luminescent center plays a key role in PersL phosphors. The commonly used luminescent centers that can generate PersL include lanthanide series, transition metal elements, and other ions such as $\mathrm{Bi}^{3+9-15}$. The latter two kinds of luminescent centers are generally limited to their relatively narrow emitting spectral bands, mainly in the visible spectral range. In contrast, the trivalent lanthanides are especially

\footnotetext{
Correspondence: Yanmin Yang (yangym@hbu.edu.cn) or

Jianrong Qiu (qj@@ju.edu.cn)

${ }^{1}$ Hebei Key Laboratory of Optic-electronic Information and Materials, College of Physics Science \& Technology, Hebei University, Baoding 071002, China

${ }^{2}$ California NanoSystems Institute, University of California, Los Angeles, CA 90095, USA

Full list of author information is available at the end of the article These authors contributed equally: Leipeng Li, Tianyi Li, Yue Hu, Chongyang Cai
}

attractive as their $4 \mathrm{f}$ electrons are shielded by the outer $5 \mathrm{~s}$ and $5 p$ electrons and are thus less affected by the surrounding crystalline field, making the $4 \mathrm{f} \leftrightarrow 4 \mathrm{f}$ transitions feature narrow-band, high color purity and wide spectral range from the UV to $\mathrm{NIR}^{16}$. In spite of these attractive points, the previous literatures focus mainly on visible PersL of the trivalent lanthanides. To achieve PersL in the UV or even deep UV and NIR range is still a challenge, although there has been some related work ${ }^{17-20}$. Moreover, there are other problems such as how to tailor the intensity of PersL, how to obtain desirable excitation and emission bands, and so forth. Fundamentally, all these questions can be ascribed to the absence of a deep understanding on PersL or a more reasonable PersL model. Since the green phosphor of $\mathrm{SrAl}_{2} \mathrm{O}_{4}: \mathrm{Eu}^{2+}, \mathrm{Dy}^{3+}$ has been shown to own excellent PersL ${ }^{21}$, several underlying mechanisms have been put forward to explain PersL, including the hole trapping-detrapping model, the electron trapping-detrapping model, and the quantum tunneling model ${ }^{1,2,22-25}$. Although these models could explain some observed phenomena, there are flaws for these models and some key points still remain unclear. It

\section{(c) The Author(s) 2022}

(c) (i) Open Access This article is licensed under a Creative Commons Attribution 4.0 International License, which permits use, sharing, adaptation, distribution and reproduction cc) in any medium or format, as long as you give appropriate credit to the original author(s) and the source, provide a link to the Creative Commons license, and indicate if changes were made. The images or other third party material in this article are included in the article's Creative Commons license, unless indicated otherwise in a credit line to the material. If material is not included in the article's Creative Commons license and your intended use is not permitted by statutory regulation or exceeds the permitted use, you will need to obtain permission directly from the copyright holder. To view a copy of this license, visit http://creativecommons.org/licenses/by/4.0/. 
is difficult to put forward a universal mechanism to reasonably explain all experimental results, and here we mainly focus on the PersL of the trivalent lanthanides in wide bandgap hosts due to its extensive application value $^{20}$.

Here we show the trivalent lanthanides with the exception of $\mathrm{La}^{3+}, \mathrm{Lu}^{3+}$, and $\mathrm{Pm}^{3+}$ could generate PersL ranging from 200 to $1700 \mathrm{~nm}$ in the selected wide bandgap hosts $\mathrm{NaYF}_{4}, \mathrm{Cs}_{2} \mathrm{NaYF}_{6}, \mathrm{XPO}_{4}(X=\mathrm{Y}, \mathrm{Sc}, \mathrm{Lu}$, and $\mathrm{La}$ ), and $\mathrm{YBO}_{3}$. Depending on the abundant experiments and analysis, a mechanism of the trivalent lanthanides' PersL in wide bandgap hosts is proposed. It is found that the energy transfer from the excitons formed in wide bandgap materials to the trivalent lanthanides plays a key role for PersL. In addition, the trivalent lanthanides as isoelectronic traps bind excitons, and the binding ability not only depends on the inherent arrangement of $4 \mathrm{f}$ electrons of the trivalent lanthanides, but also on the extrinsic ligand field including anion coordination and cation substitution.

\section{Results}

Except for $\mathrm{La}^{3+}$ without the $4 \mathrm{f}$ electrons and $\mathrm{Lu}^{3+}$ with full filled $4 \mathrm{f}$ shell and radioactive $\mathrm{Pm}^{3+}$, the rest trivalent lanthanides' PersL was observed with success in the selected hosts $\mathrm{NaYF}_{4}, \mathrm{Cs}_{2} \mathrm{NaYF}_{6}, \mathrm{YPO}_{4}$, and $\mathrm{ScPO}_{4}$ (Fig. 1a, Supplementary Fig. S1). The wavelength of these PersL bands ranges broadly from 200 to $1700 \mathrm{~nm}$ (Fig. 1b). The whole wavelength range of the PersL could be divided into the UV, visible, and NIR three parts. Most of the trivalent lanthanides, excluding $\mathrm{Gd}^{3+}$ and $\mathrm{Yb}^{3+}$, emitted PersL in the visible spectral range. It can be seen that the decay time of the visible PersL is relatively long (Fig. 1c). The shortest decay time originating from the $542 \mathrm{~nm}$ emission line of $\mathrm{Ho}^{3+}$ and the $542 \mathrm{~nm}$ counterpart of $\mathrm{Er}^{3+}$ has exceeded $40 \mathrm{~h}$. Notably, the green PersL attributed to the ${ }^{5} \mathrm{D}_{4} \rightarrow{ }^{7} \mathrm{~F}_{5}$ transition of $\mathrm{Tb}^{3+}$ could be collected with a recognizable signal-to-noise ratio even after $200 \mathrm{~h}$. In the wavelength range of $760-1700 \mathrm{~nm}$, $\mathrm{Nd}^{3+}, \mathrm{Ho}^{3+}$, and $\mathrm{Er}^{3+}$ emitted the NIR PersL, and their decay time could last for at least $3 \mathrm{~h}$, making these three ions possible luminescent markers for medical imaging (Fig. 1d). The above-mentioned eight PersL bands were clearly measured by spectrometer even after several days (Supplementary Fig. S2). The PersL belonging to the ${ }^{6} \mathrm{P}_{7 / 2} \rightarrow{ }^{8} \mathrm{~S}_{7 / 2}$ transition of $\mathrm{Gd}^{3+}$ in $\mathrm{ScPO}_{4}$ was also detected directly for the first time, to the best of our knowledge (Fig. 1a, e). It should be mentioned here that Pan's group has recently also reported the PersL behavior of this transition, which is, however, on the basis of energy

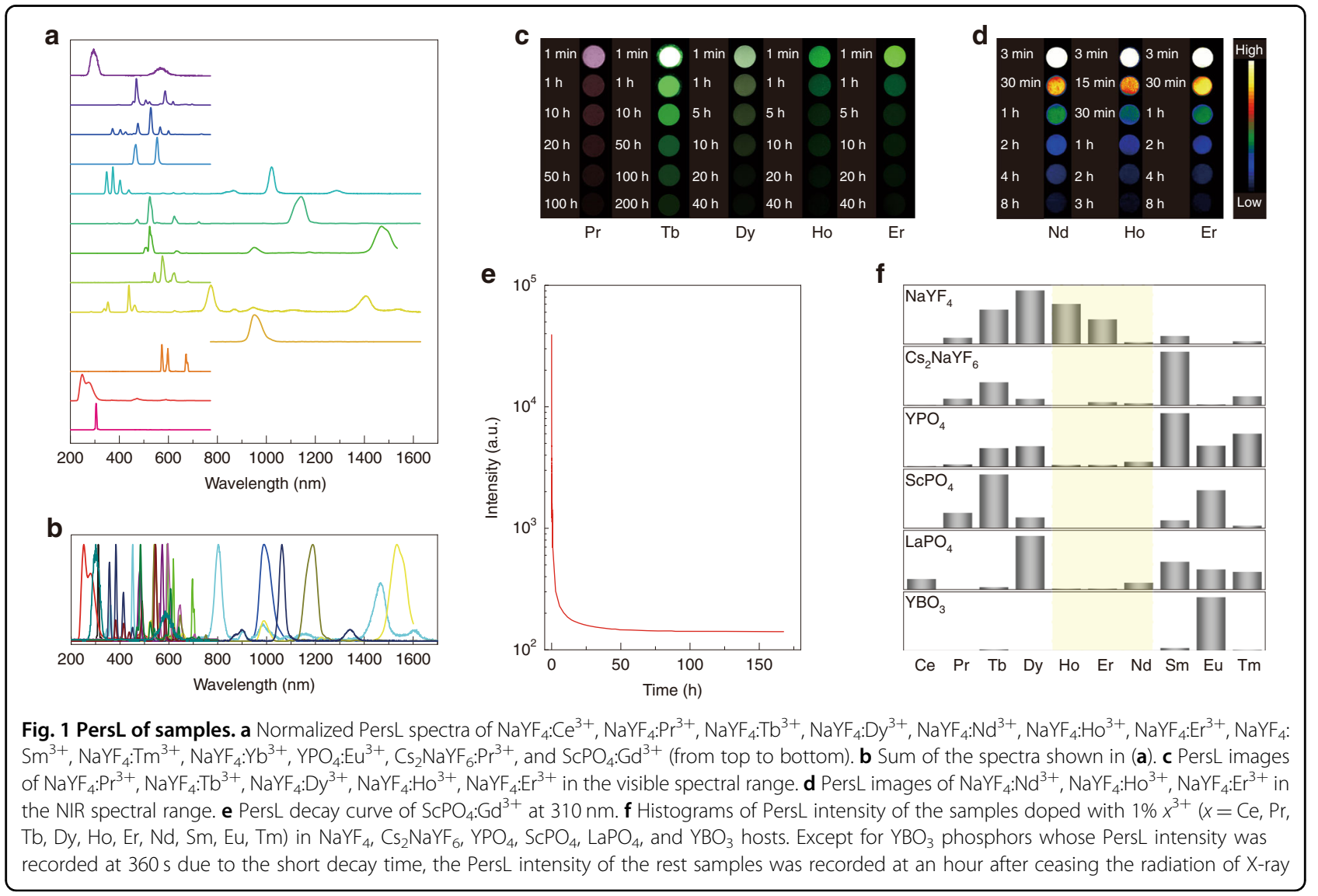



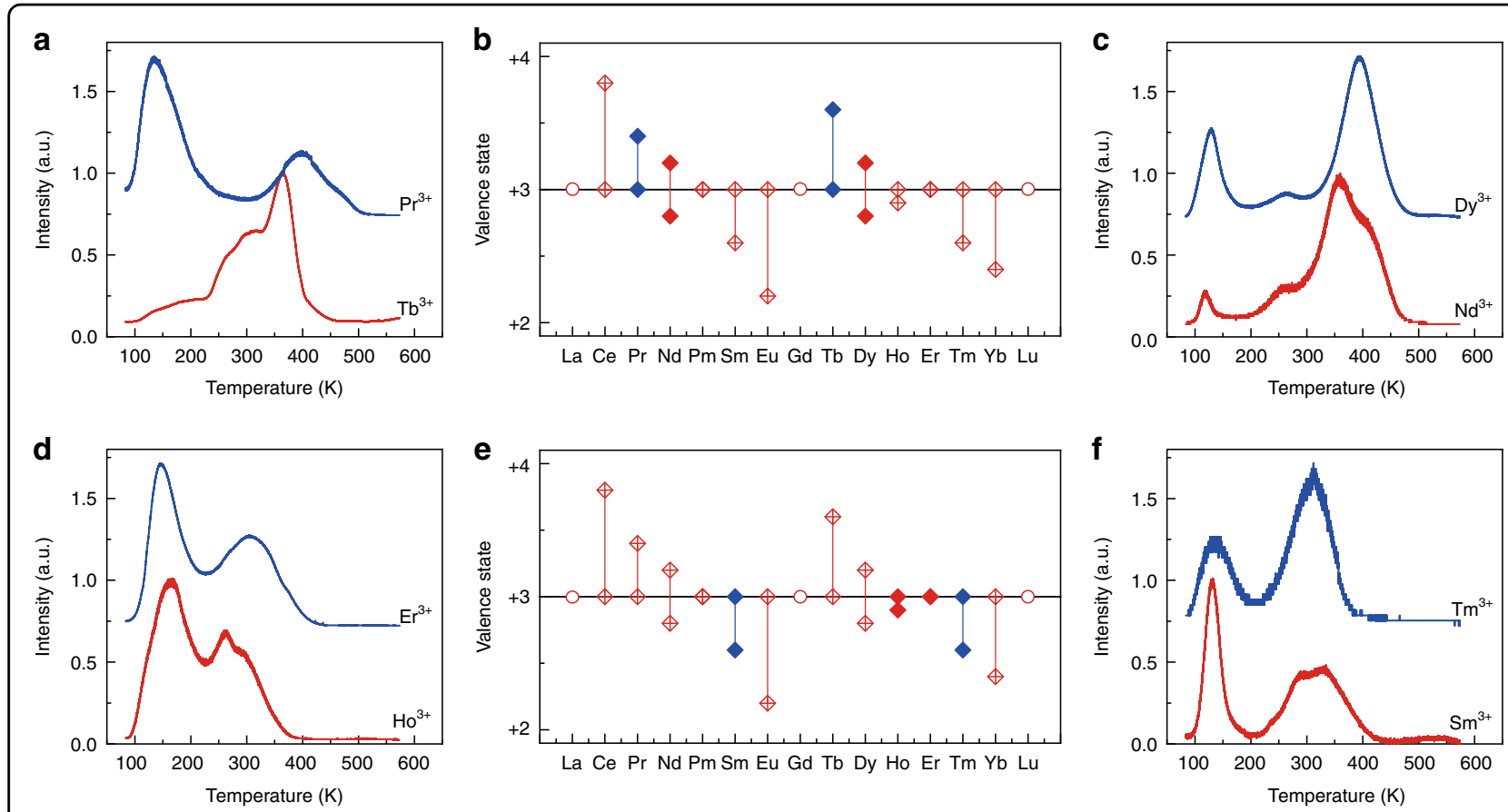

Fig. 2 TLs of the trivalent lanthanides in $\mathrm{NaYF}_{4}$. a TL curves by monitoring the $608 \mathrm{~nm}$ line of $\mathrm{Pr}^{3+}$ and the $543 \mathrm{~nm}$ line of $\mathrm{Tb}^{3+}$. c $\mathrm{TL}_{\mathrm{Curves}}$ by monitoring the $571 \mathrm{~nm}$ line of $\mathrm{Dy}^{3+}$ and the $382 \mathrm{~nm}$ line of $\mathrm{Nd}^{3+}$. d TL curves by monitoring the $542 \mathrm{~nm}$ line of $\mathrm{Er}^{3+}$ and the $542 \mathrm{~nm}$ line of $\mathrm{Ho}^{3+}$. f TL curves by monitoring the $451 \mathrm{~nm}$ line of $\mathrm{Tm}^{3+}$ and the $594 \mathrm{~nm}$ line of $\mathrm{Sm}^{3+}$. b e Variation trend of the valence state of the trivalent lanthanides

transfer from other luminescent centers to $\mathrm{Gd}^{3+26}$. The decay time of the PersL of $\mathrm{Gd}^{3+}$ surpasses $170 \mathrm{~h}$, one of the longest decay times known so far. In addition to $\mathrm{Gd}^{3+}$, $\mathrm{Pr}^{3+}$ also presented PersL in the UV range peaking at $\sim 251 \mathrm{~nm}$, the shortest wavelength of all the samples reported here (Supplementary Fig. S3).

After ceasing the $\mathrm{X}$-ray source, the PersL of the trivalent lanthanides in the hosts $\mathrm{NaYF}_{4}, \mathrm{Cs}_{2} \mathrm{NaYF}_{6}, \mathrm{YPO}_{4}, \mathrm{ScPO}_{4}$, $\mathrm{LaPO}_{4}$, and $\mathrm{YBO}_{3}$ displays different decay behaviors (Fig. 1f, Supplementary Fig. S4-S15). The PersL of $\mathrm{Er}^{3+}$, $\mathrm{Ho}^{3+}$, and $\mathrm{Nd}^{3+}$ is nearly undetectable in the hosts $\mathrm{Cs}_{2} \mathrm{NaYF}_{6}, \mathrm{YPO}_{4}, \mathrm{ScPO}_{4}, \mathrm{LaPO}_{4}$, and $\mathrm{YBO}_{3}$, as well as in other hosts such as silicate, aluminate, and zincate (not shown herein). In contrast, $\mathrm{Er}^{3+}, \mathrm{Ho}^{3+}$, and $\mathrm{Nd}^{3+}$ emitted considerable PersL that could last for at least $10 \mathrm{~h}$ in $\mathrm{NaYF}_{4}$. Moreover, the PersL spectra of $\mathrm{Er}^{3+}, \mathrm{Nd}^{3+}$, and $\mathrm{Ho}^{3+}$ in $\mathrm{Cs}_{2} \mathrm{NaYF}_{6}, \mathrm{YPO}_{4}, \mathrm{ScPO}_{4}, \mathrm{LaPO}_{4}$, and $\mathrm{YBO}_{3}$ are different from that in $\mathrm{NaYF}_{4}$ (Supplementary Fig. S16). For instance, $\mathrm{Er}^{3+}$ generally emits green and red luminescence which is attributed to the ${ }^{2} \mathrm{H}_{11 / 2} /{ }^{4} \mathrm{~S}_{3 / 2} \rightarrow{ }^{4} \mathrm{I}_{15 / 2}$ and ${ }^{4} \mathrm{~F}_{9 / 2} \rightarrow{ }^{4} \mathrm{I}_{15 / 2}$ transitions in the $200-800 \mathrm{~nm}$ wavelength range, as shown in the top PersL spectrum of Supplementary Fig. S16a for $\mathrm{NaYF}_{4}: \mathrm{Er}^{3+27-29}$. Nonetheless, the green and red emissions of $\mathrm{Er}^{3+}$ in $\mathrm{Cs}_{2} \mathrm{NaYF}_{6}$, $\mathrm{YPO}_{4}, \mathrm{ScPO}_{4}, \mathrm{LaPO}_{4}$, and $\mathrm{YBO}_{3}$ are seriously weakened. And the transitions with a higher energy than ${ }^{2} \mathrm{H}_{11 / 2} /{ }^{4} \mathrm{~S}_{3 / 2}$ states dominate the emissions. The different emitting characteristics of $\mathrm{Er}^{3+}, \mathrm{Nd}^{3+}$, and $\mathrm{Ho}^{3+}$ in different hosts are expected to be responsible for their different PersL behaviors, which will be further discussed in the following. Thermoluminescence (TL) of the prepared samples was also measured (Fig. 2). As can be observed, the positions of the TL peaks of $\mathrm{Pr}^{3+}, \mathrm{Dy}^{3+}, \mathrm{Er}^{3+}$, and $\mathrm{Tm}^{3+}$ correspond roughly to that of $\mathrm{Tb}^{3+}, \mathrm{Nd}^{3+}, \mathrm{Ho}^{3+}$, and $\mathrm{Sm}^{3+}$ (Fig. 2a, c, d, f), which is in good agreement with the variation trend of the valence state of the trivalent lanthanides (Fig. 2b, e) ${ }^{2}$.

\section{Discussion}

\section{Exciton and attractive potential}

The band-to-band excitation is an important and necessary charging way for wide bandgap materials embedded with the trivalent lanthanides ${ }^{2}$. Our samples, due to their wide bandgap, cannot be charged by the excitation source whose energy is below the host's bandgap such as mercury lamp. As demonstrated before, excitons exist in wide bandgap materials at room temperature as the binding energy of exciton is proportional to the bandgap of host (Supplementary Fig. S17) ${ }^{30-32}$. In order to generate PersL, the trivalent lanthanides, as isoelectronic trap when substituting the trivalent cations in wide bandgap host, are expected to have the ability to bind excitons to get their recombination energy. According to the literature ${ }^{33}$, the ability of binding carriers for the trivalent lanthanides which is defined as the attractive potential $V$, is mainly determined by the 
intrinsic arrangement of the $4 \mathrm{f}$ electrons. Here we show this ability is also associated with the extrinsic ligand field including anion coordination and cation substitution:

$$
V=V_{O}+V_{A}+V_{C}
$$

where $V_{O}, V_{A}$, and $V_{C}$ are the attractive potentials aroused by the arrangement of the $4 \mathrm{f}$ electrons of the trivalent lanthanides, anion coordination and cation substitution, respectively.

\section{Intrinsic: arrangement of the $4 \mathrm{f}$ electrons}

This topic is extended herein from three perspectives. First, the double-double effect summarized by Fidelis et al. ${ }^{34}$ indicates that the electron configuration with even total orbital angular momenta is relatively stable (Supplementary Table S1), including $\mathrm{La}^{3+}, \mathrm{Gd}^{3+}, \mathrm{Lu}^{3+}, \mathrm{Er}^{3+}$, $\mathrm{Nd}^{3+}, \mathrm{Ho}^{3+}, \mathrm{Pm}^{3+}$. By comparison, the trivalent lanthanides with odd total orbital angular momenta, that are, $\mathrm{Ce}^{3+}, \mathrm{Tb}^{3+}, \mathrm{Pr}^{3+}, \mathrm{Dy}^{3+}, \mathrm{Eu}^{3+}, \mathrm{Sm}^{3+}, \mathrm{Tm}^{3+}$, and $\mathrm{Yb}^{3+}$, are not such stable. It reveals that the stability of $\mathrm{Er}^{3+}, \mathrm{Nd}^{3+}$, $\mathrm{Ho}^{3+}$, and $\mathrm{Pm}^{3+}$ is close to that of $\mathrm{La}^{3+}, \mathrm{Gd}^{3+}$, and $\mathrm{Lu}^{3+}$. As clearly presented in Fig. 3a, the whole trivalent lanthanides can be grouped into $\left[\mathrm{Ce}^{3+}, \mathrm{Pr}^{3+}, \mathrm{Tb}^{3+}, \mathrm{Dy}^{3+}\right.$,
$\mathrm{Sm}^{3+}, \mathrm{Eu}^{3+}, \mathrm{Tm}^{3+}$, and $\left.\mathrm{Yb}^{3+}\right]$ and $\left[\mathrm{Er}^{3+}, \mathrm{Nd}^{3+}, \mathrm{Ho}^{3+}\right.$, $\mathrm{Pm}^{3+}, \mathrm{La}^{3+}, \mathrm{Gd}^{3+}$, and $\mathrm{Lu}^{3+}$, which agrees well with the order of stability. Second, the variation trend of valence state suggests obviously that $\mathrm{La}^{3+}, \mathrm{Gd}^{3+}$, and $\mathrm{Lu}^{3+}$ are the most stable trivalent lanthanides, followed by $\mathrm{Er}^{3+}, \mathrm{Nd}^{3+}$, $\mathrm{Ho}^{3+}$ and $\mathrm{Pm}^{3+}$ (Fig. 3b) $)^{2} \cdot \mathrm{Ce}^{3+}, \mathrm{Pr}^{3+}, \mathrm{Tb}^{3+}$, and $\mathrm{Dy}^{3+}$ are inclined to lose electron to be quadrivalent while $\mathrm{Sm}^{3+}$, $\mathrm{Eu}^{3+}, \mathrm{Tm}^{3+}$, and $\mathrm{Yb}^{3+}$ tend to gain electron to be bivalent. It can be concluded that the result in Fig. $3 \mathrm{~b}$ is almost identical to the law presented in Fig. 3a. The major difference is that the double-double effect is unidirectional while the variation trend of valence state is bidirectional. So the undetectable PersL of $\mathrm{Er}^{3+}, \mathrm{Nd}^{3+}$, and $\mathrm{Ho}^{3+}$ in oxysalts is explained by the fact that it is difficult for them to bind carries (Fig. 1f). The similar TL curves (Fig. 2a, c, d, f) of four groups of the trivalent lanthanides could also be reasonably ascribed to the similar carrierbinding ability. Third, the energy difference between the lowest energy level of the $4 \mathrm{f}$ electron configuration of the trivalent lanthanides and that of $\mathrm{Ce}^{3+}$ shows the similar results with the above-mentioned two evidences (Fig. 3c) ${ }^{35}$. On the whole, the trivalent lanthanides can be roughly divided into four groups, $\left[\mathrm{Ce}^{3+}, \mathrm{Pr}^{3+}, \mathrm{Tb}^{3+}\right.$, $\left.\mathrm{Dy}^{3+}\right],\left[\mathrm{Sm}^{3+}, \mathrm{Eu}^{3+}, \mathrm{Tm}^{3+}, \mathrm{Yb}^{3+}\right],\left[\mathrm{Er}^{3+}, \mathrm{Nd}^{3+}, \mathrm{Ho}^{3+}\right.$,
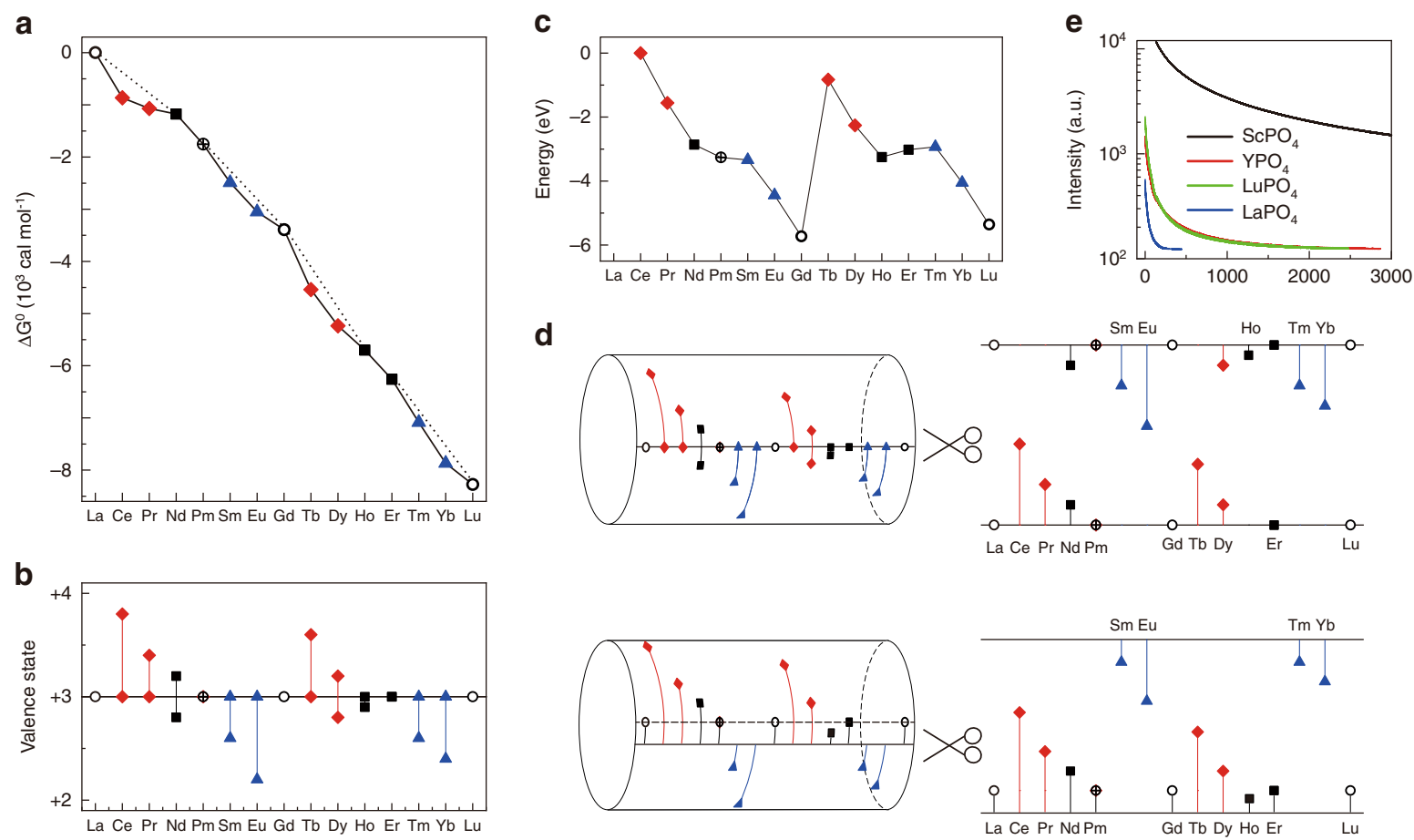

Fig. 3 Modulation of the trivalent lanthanides' attractive potential. a Free energy changes $\left(\Delta G^{0}\right)$ of the trivalent lanthanides complexes with 2-ethylhexyl phenylphosphonic acid complexing agent relative to that of $\mathrm{La}^{3+}$. Reprinted from ref. ${ }^{34}$, Copyright 1976, with permission from Elsevier. b Variation trend of the valence state of the trivalent lanthanides. The length of each vertical line shows the relative ability of changing the valence state. $\mathbf{c}$ Energy difference between the lowest energy level of the $4 \mathrm{f}$ electron configuration of the trivalent lanthanides and that of $\mathrm{Ce}^{3+}$. Reprinted from ref. ${ }^{35}$, Copyright 2009, with permission from Elsevier. $\mathbf{d}$ Schematic diagram of modulating the position of the trivalent lanthanides in the forbidden band. e PersL decay cures of $\mathrm{Gd}^{3+}$ doped $\mathrm{XPO}_{4}(X=\mathrm{Sc}, \mathrm{Y}, \mathrm{La}, \mathrm{Lu})$ 
$\left.\mathrm{Pm}^{3+}\right]$, and $\left[\mathrm{La}^{3+}, \mathrm{Gd}^{3+}, \mathrm{Lu}^{3+}\right]$, although there might be some little discrepancy.

All these evidences indicate undoubtedly that the attractive potential of the trivalent lanthanides at their free state is mainly determined by the intrinsic arrangement of the $4 \mathrm{f}$ electrons, which gives us a clear figure of the ability of binding carriers for the trivalent lanthanides in wide bandgap materials. It's worth noting that the zigzag model cannot be used to explain our findings here, especially the ultralong PersL of phosphors doped with $\mathrm{Gd}^{3+}$. Gd is always in +3 valence outside of several exotic compounds. Therefore, we cannot use the core point of the zig-zag model, i.e., valence alternation, to account for the ultralong PersL of phosphors doped with $\mathrm{Gd}^{3+}$. More importantly, the ground state of $\mathrm{Gd}^{2+}$ is always above the conduction band edge and that of $\mathrm{Gd}^{3+}$ will be firmly below the valence band edge, and the so-called "bandgap engineering" does not change the fact. In light of the zigzag model, the PersL of phosphors doped with $\mathrm{Gd}^{3+}$ should never occur, which is obviously against our findings. So the zig-zag model is not the substance of the PersL of the trivalent lanthanides in wide bandgap materials, although it sometimes reflects similar trends. At this point, it should be emphasized that the above discussions are only suitable for free trivalent lanthanides, meaning that they only reflect the property of the intrinsic arrangement of the $4 \mathrm{f}$ electrons. In addition to this "internal" factor, the "external" aspects, mainly including anion coordination and cation substitution, have also an effect on the ability of binding carriers of the trivalent lanthanides in crystals, as discussed below.

\section{Extrinsic I: anion coordination}

In ionic crystals such as $\mathrm{NaYF}_{4}$, the valence electrons of the trivalent lanthanides are completely occupied by the coordination anions. The attractive potential of the trivalent lanthanides' $4 \mathrm{f}$ electrons is thus less affected by the shielding effect of the valence electrons. The effect of electron cloud polarization becomes appreciable, making there an additional ability on the original attractive potential of the trivalent lanthanides' $4 \mathrm{f}$ electrons. Therefore, $\mathrm{Er}^{3+}, \mathrm{Ho}^{3+}$, and $\mathrm{Nd}^{3+}$ that are not easy to bind charges also own the ability to bind carriers to generate emission upon band-to-band excitation. In phosphates and borates hosts, the interaction between the trivalent lanthanides and coordination anions changes from ionic to covalent. The increase in the electrostatic shielding effect of valence electrons on the trivalent lanthanides' $4 \mathrm{f}$ electrons results in a decrease of their attractive potential. As mentioned above, $\mathrm{Er}^{3+}, \mathrm{Ho}^{3+}$, and $\mathrm{Nd}^{3+}$ in their free state have weak ability to bind charge carriers, and this ability has been further weakened in covalent crystals. This probably explains the missing PersL of $\mathrm{Er}^{3+}, \mathrm{Ho}^{3+}$, and $\mathrm{Nd}^{3+}$ in phosphates and borates (Fig. 1f).

\section{Extrinsic II: cation substitution}

In addition to the strategy of anion coordination, cation substitution is also commonly utilized to adjust crystalline field. When substituting the trivalent lanthanides for the trivalent host cations, the isoelectronic traps are formed, followed by the generation of bound state by a short-range central-cell potential. According to the reported work ${ }^{36-39}$, the primary factor affecting the binding potential of the isoelectronic traps is electronegativity and size difference between the impurity and host ions. Therefore, the difference of electronegativity and the size difference between the substitution ion and the trivalent lanthanides make there a bound state for the trivalent lanthanides in the forbidden band. The attractive potential and position of the trivalent lanthanides in the forbidden band are thus adjustable by changing the cation substitution (Fig. 3d). It reminds us that the PersL of the trivalent lanthanides can be expected, adjusted, and even improved via a rational design of the external factor of cation substitution. It has been verified by the samples of $\mathrm{Gd}^{3+}$ doped $X \mathrm{PO}_{4}(X=\mathrm{Sc}$, $\mathrm{Y}, \mathrm{La}, \mathrm{Lu}$ ) (Fig. 3e). The longest PersL of $\mathrm{Gd}^{3+}$ is achieved in $\mathrm{ScPO}_{4}$ because $\mathrm{Sc}^{3+}$, compared with $\mathrm{Lu}^{3+}, \mathrm{Y}^{3+}$, and $\mathrm{La}^{3+}$, has a larger difference in electronegativity and size with $\mathrm{Gd}^{3+}$. The similar conclusion was also observed in the samples of $\mathrm{Gd}^{3+}$ doped $X_{\mathrm{BO}_{3}}(X=\mathrm{Sc}, \mathrm{Y}, \mathrm{La}, \mathrm{Lu})$ (Supplementary Fig. S18). The larger the difference between $\mathrm{Gd}^{3+}$ and substitution ion, the longer the PersL decay time of $\mathrm{Gd}^{3+}$, in good accordance with the above-mentioned hypothesis.

\section{PersL mechanism of the trivalent lanthanides}

Depending on the above-mentioned discussions, the possible mechanism of the trivalent lanthanides' PersL in wide bandgap hosts is proposed (top panel in Fig. 4). Upon excitation of X-ray, the electrons jump from the valence band to the conduction band, leaving there a large amount of holes in the valence band (1). Subsequently, these electrons and holes are captured by the traps (2). After ceasing the X-ray source, the captured electrons and holes are released from the traps to the conduction band and valence band, respectively, due to the stimulation of heat (3). The trivalent lanthanides attract an electron (or hole) first and then draw a hole (or electron) due to the Coulomb force (4), 5), case 1 and case 2). It should be emphasized there is the possibility that the released electrons and holes attract each other to form excitons. Due to the electrically neutral characteristic, the excitons migrate among the crystal lattice and are finally captured by the trivalent lanthanides (5). In all cases, the trivalent lanthanides are expected to finally bound the excitons and then obtain the recombination energy of the bounded excitons to jump to the excited states (6) ${ }^{11}$. After nonradiative relaxation processes, the PersL occurs due to the 4f $\leftrightarrow 4 \mathrm{f}$ transition of the trivalent lanthanides (6). 


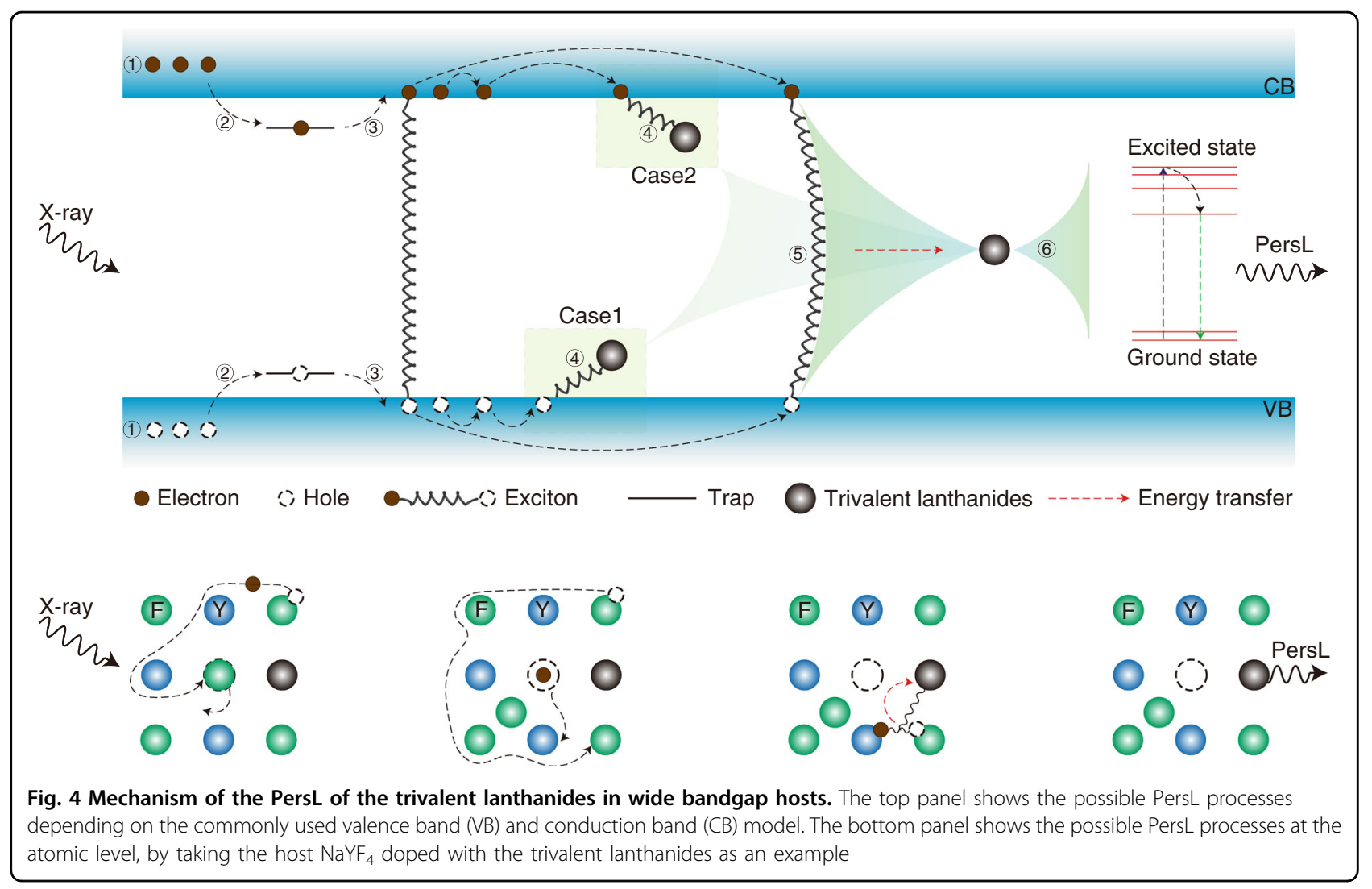

To further understand the specific generation process of PersL, the possible mechanism is then discussed at the atomic level (bottom panel in Fig. 4), by taking the host $\mathrm{NaYF}_{4}$ embedded with the trivalent lanthanides as an example. For $\mathrm{NaYF}_{4}$, the top of the valence band and the bottom of the conduction band separately belong to the $2 \mathrm{p}$ electron orbit of $\mathrm{F}$ and the $5 \mathrm{~d}$ electron orbit of $\mathrm{Y}$. The isoelectronic cation substitution, namely replacing the $\mathrm{Y}^{3+}$ in $\mathrm{NaYF}_{4}$ with other trivalent lanthanides, forms bound states by a short-range central-cell potential. Upon excitation of X-ray, the outer electrons of $\mathrm{F}$ are excited to the $5 \mathrm{~d}$ electron orbit of $\mathrm{Y}$, while the generated holes stay in F. Upon X-ray irradiation, the F ions are likely to be ejected from their original sites, making there many fluorine vacancies and interstitial $\mathrm{F}$ that act as traps ${ }^{29}$. The electrons in the conduction band and the holes in the valence band are thus trapped with success. After ceasing the X-ray source, the electrons are released from the defects to the $5 \mathrm{~d}$ electron orbit of $\mathrm{Y}$ due to the stimulation of heat. The trivalent lanthanides are expected to attract an electron (or hole) first and then will draw a hole (or electron) due to the Coulomb force. It should be mentioned that the released electrons and holes are likely to attract each other to form excitons that migrate easily among the crystal lattice because of the electrically neutral feature. In all cases, the trivalent lanthanides eventually bind the excitons as this is the origin of energy. The excitons recombine and transfer the energy to the trivalent lanthanides that bind the excitons. The trivalent lanthanides are excited from the ground state to the excited state, which is followed by the non-radiative relaxation processes and final emitting of PersL.

\section{Conclusion}

In summary, the PersL of the trivalent lanthanides with the exception of $\mathrm{Lu}^{3+}, \mathrm{La}^{3+}$, and $\mathrm{Pm}^{3+}$ covering a broad wavelength range from 200 to $1700 \mathrm{~nm}$ are observed in several wide bandgap materials, upon the band-to-band charging way via X-ray irradiation. A mechanism of the trivalent lanthanides' PersL in wide bandgap hosts is proposed. According to the mechanism, the trivalent lanthanides as isoelectronic traps are expected to eventually bind excitons, and this binding ability is not only related to the inherent arrangement of the $4 \mathrm{f}$ electrons of the trivalent lanthanides, but also to the extrinsic anion coordination and cation substitution. The excitons in wide bandgap materials transfer their recombination energy to the trivalent lanthanides, followed by the generation of PersL from the trivalent lanthanides. Guided by this mechanism, the direct ultralong PersL of $\mathrm{Gd}^{3+}$ is achieved for the first time in phosphates, proving the validity of our proposed mechanism. Our work not only 
widens the range of the trivalent lanthanides activated PersL phosphors but also proposes a mechanism with considerable rationality, which is believed to be a guidance for designing high-performance and more ideal PersL phosphors in the future.

\section{Materials and methods \\ Fabrication of samples}

All PersL phosphors were prepared by hightemperature solid-state method and the doping concentration of the trivalent lanthanides was set to be $1 \%$ mol. NaF (Aladdin, 99.99\%), $\mathrm{NH}_{4} \mathrm{~F}$ (Aladdin, 99.99\%), $X_{2} \mathrm{O}_{3}(X=\mathrm{Y}, \mathrm{La}, \mathrm{Ce}, \mathrm{Nd}, \mathrm{Sm}, \mathrm{Eu}, \mathrm{Gd}$, Dy, Ho, Er, Tm, and $\mathrm{Yb}$, Aladdin, 99.99\%), $\mathrm{Pr}_{6} \mathrm{O}_{11}$ (Aladdin, 99.99\%), $\mathrm{Tb}_{4} \mathrm{O}_{7}$ (Aladdin, 99.99\%), $\mathrm{Cs}_{2} \mathrm{CO}_{3}$ (Aladdin, 99.99\%), $\mathrm{NaHCO}_{3}$ (Aladdin, 99.99\%), $\mathrm{NH}_{4} \mathrm{H}_{2} \mathrm{PO}_{4}$ (Aladdin, 99.95\%), $\mathrm{Sc}_{2} \mathrm{O}_{3}$ (Aladdin, 99.99\%), and $\mathrm{H}_{3} \mathrm{BO}_{3}$ (Aladdin, 99.99\%) were used as raw materials. The general preparation processes for the samples are described as follows. The stoichiometric raw materials were weighed and mixed well in mortar to form homogeneous powders. These powders were then transferred into aluminum oxide crucible to be calcined in muffle furnace at the given temperature for several hours. Finally, the obtained powders were cooled down to room temperature to form the final phosphors. To form $\mathrm{NaYF}_{4}$ phosphors, the homogeneous powders were calcined at $500^{\circ} \mathrm{C}$ for $2 \mathrm{~h}$. For the preparation of $\mathrm{Cs}_{2} \mathrm{NaYF}_{6}$, the homogeneous powders were pre-fired at $150^{\circ} \mathrm{C}$ in air for $7 \mathrm{~h}$, and were then sintered at $450{ }^{\circ} \mathrm{C}$ for $30 \mathrm{~min}$, followed by the final calcination at $700^{\circ} \mathrm{C}$ for $10 \mathrm{~h}$ under nitrogen atmosphere. To obtain $\mathrm{YPO}_{4}, \mathrm{ScPO}_{4}$, and $\mathrm{LaPO}_{4}$ samples, the homogeneous raw materials should be calcined at $500^{\circ} \mathrm{C}$ for $2 \mathrm{~h}$ under nitrogen atmosphere first and then at $1300^{\circ} \mathrm{C}$ for $5 \mathrm{~h}$. To get $\mathrm{YBO}_{3}$ phosphors, the raw powders were annealed under nitrogen atmosphere at $500{ }^{\circ} \mathrm{C}$ for $1 \mathrm{~h}$ first and then at $1300^{\circ} \mathrm{C}$ for $2 \mathrm{~h}$. It was demonstrated that the samples were micro-sized with irregular shape (Supplementary Figs. S19-S24). In addition, these phosphors were of pure phase (Supplementary Fig. S25).

\section{Charging of samples}

The PersL phosphors were charged by the X-ray source of XRad-320X-ray irradiator (Precision X-ray, Inc., North Branford, CT) equipped with a tungsten target $(40 \mathrm{kV}$, $30 \mathrm{~mA}$ ). Before measurement on the property of PersL, all samples were irradiated by X-ray for $10 \mathrm{~min}$ to be charged.

\section{Photoluminescence (PL) and PersL characterization of samples}

The PL spectra of samples upon X-ray excitation, PersL spectra, and decay curves of PersL in the visible light band were recorded using an Andor SR-500i spectrometer
(Andor Technology Co. Belfast, UK) equipped with a Hamamatsu R928 photomultiplier. The visible PersL images were recorded using a digital SLR camera (EOS 5D Mark III) in darkroom. The NIR PersL images were recorded using a CCD camera (DU-888U3-CS0-BV).

\section{TL characterization of samples}

TL measurements were carried out using a selfassembling TL system including high precision thermal stage (THMS600) (British Linkam Scientific Instruments) and a Andor SR-500i spectrometer (Andor Technology Co., Belfast, UK), with a fixed heating rate of $5 \mathrm{~K} / \mathrm{s}$ within the range of $83-600 \mathrm{~K}$. The samples were irradiated by $\mathrm{X}$-ray for $10 \mathrm{~min}$ before TL measurement.

\section{Vacuum ultraviolet (VUV) excitation characterization of samples}

The VUV excitation spectra were measured at the VUV spectroscopy experimental station on beam line 4B8 of Beijing Synchrotron Radiation Facility (BSRF). The excitation and emission spectra were measured by using a $1 \mathrm{~m}$ Seya monochromator $\left(1200 \mathrm{~g} \cdot \mathrm{mm}^{-1}, 120-350 \mathrm{~nm}, 1 \mathrm{~nm}\right.$ bandwidth) and an Acton SP-308 monochromator $\left(600 \mathrm{~g} \cdot \mathrm{mm}^{-1}, 330-900 \mathrm{~nm}\right)$. The signal was detected by a Hamamatsu H8259-01 photon-counting unit and corrected by the excitation intensity of sodium salicylate $\left(\mathrm{o}-\mathrm{C}_{6} \mathrm{H}_{4} \mathrm{OHCOONa}\right)$ measured simultaneously under the same condition.

\section{Acknowledgements}

L.L. thanks the support from the Advanced Talents Incubation Program of Hebei University (521100221006). Y.Y. acknowledges the financial support from the National Natural Science Foundation of China (11974097) and the Natural Science Foundation of Hebei Province (A2019201073). J.Q. acknowledges the support from the National Natural Science Foundation of China (52161135109).

\section{Author details}

${ }^{1}$ Hebei Key Laboratory of Optic-electronic Information and Materials, College of Physics Science \& Technology, Hebei University, Baoding 071002, China. ${ }^{2}$ California NanoSystems Institute, University of California, Los Angeles, CA 90095, USA. ${ }^{3}$ State Key Laboratory of Modern Optical Instrumentation, College of Optical Science and Engineering, Zhejiang University, Hangzhou 310058, China

\section{Author contributions}

Y.Y. and J.Q. conceived the project. L.L. analyzed data and mainly wrote the paper. T.L., Y.H., and C.C. synthesized the phosphors and conducted spectral measurements. T.L. and B.L. carried out the NIR imaging experiments. All of the authors discussed the results and contributed to the writing of the paper.

Conflict of interest

The authors declare no competing interests.

Supplementary information The online version contains supplementary material available at https://doi.org/10.1038/s41377-022-00736-5.

Received: 19 December 2021 Revised: 28 January 2022 Accepted: 11 February 2022

Published online: 08 March 2022 


\section{References}

1. Xu, J. \& Tanabe, S. Persistent luminescence instead of phosphorescence: History, mechanism, and perspective. J. Lumin. 205, 581-620 (2019).

2. Li, Y., Geceviciusa, M. \& Qiu, J. R. Long persistent phosphors-from fundamentals to applications. Chem. Soc. Rev. 45, 2090-2136 (2016).

3. Zou, X. J. et al. X-ray-induced nanoparticle-based photodynamic therapy of cancer. Nanomedicine 9, 2339-2351 (2014).

4. Sun, S. K., Wang, H. F. \& Yan, X. P. Engineering persistent luminescence nanoparticles for biological applications: from biosensing/bioimaging to theranostics. Acc. Chem. Res. 51, 1131-1143 (2018).

5. Van Den Eeckhout, K., Poelman, D. \& Smet, P. F. Persistent luminescence in non-Eu ${ }^{2+}$-doped compounds: a review. Materials 6, 2789-2818 (2013).

6. Poelman, D. et al. Persistent phosphors for the future: fit for the right application. J. Appl. Phys. 128, 240903 (2020)

7. Zhuang, Y. X. et al. X-ray-charged bright persistent luminescence in NaYF $\mathrm{Ln}^{3+} @ \mathrm{NaYF}_{4}$ nanoparticles for multidimensional optical information storage. Light.: Sci. Appl. 10, 132 (2021).

8. Liu, J. H. et al. Imaging and therapeutic applications of persistent luminescence nanomaterials. Adv. Drug Deliv. Rev. 138, 193-210 (2019).

9. Katayama, Y. et al. The role of $\mathrm{Ln}^{3+}(\mathrm{Ln}=\mathrm{Eu}, \mathrm{Yb})$ in persistent red luminescence in $\mathrm{MgGeO}_{3}: \mathrm{Mn}^{2+}$. J. Mater. Chem. C. 5, $8893-8900$ (2017).

10. Lyu, T. H. \& Dorenbos, P. Towards information storage by designing both electron and hole detrapping processes in bismuth and lanthanide-doped LiRE(Si, Ge)O $\mathrm{O}_{4}(\mathrm{RE}=\mathrm{Y}$, Lu) with high charge carrier storage capacity. Chem. Eng. J. 400, 124776 (2020)

11. Schipper, W. J. \& Blasse, G. On the recombination mechanism in X-ray storage phosphors based on lanthanum fluoride. J. Lumin. 59, 377-383 (1994).

12. Liu, F., Liang, Y. J. \& Pan, Z. W. Detection of up-converted persistent luminescence in the near infrared emitted by the $\mathrm{Zn}_{3} \mathrm{Ga}_{2} \mathrm{GeO}_{8}: \mathrm{Cr}^{3+}, \mathrm{Yb}^{3+}, \mathrm{Er}^{3+}$ phosphor. Phys. Rev. Lett. 113, 177401 (2014).

13. Chen, X. Z. et al. Trap energy upconversion-like near-infrared to near-infrared light rejuvenateable persistent luminescence. Adv. Mater. 33, 2008722 (2021)

14. Maldiney, T. et al. The in vivo activation of persistent nanophosphors for optical imaging of vascularization, tumours and grafted cells. Nat. Mater. 13 418-426 (2014)

15. Khanin, V. M. et al. Influence of $3 d$ transition metal impurities on garnet scintillator afterglow. Cryst. Growth Des. 20, 3007-3017 (2020).

16. Blasse, G. \& Grabmaier, B. C. Luminescent Materials, 232 (Springer, 1994).

17. Yang, Y. M. et al. X-ray-activated long persistent phosphors featuring strong UVC afterglow emissions. Light.: Sci. Appl. 7, 88 (2018).

18. Wang, X. L. et al. Solar-blind ultraviolet-C persistent luminescence Phosphors. Nat. Commun. 11, 2040 (2020)

19. Liang, Y. J. et al. Extending the applications for lanthanide ions: efficient emitters in short-wave infrared persistent luminescence. J. Mater. Chem. C. 5 6488-6492 (2017).
20. Pei, P. et al. X-ray-activated persistent luminescence nanomaterials for NIR-I imaging. Nat. Nanotechnol. 16, 1011-1018 (2021).

21. Matsuzawa, T. et al. A new long phosphorescent phosphor with high brightness, $\mathrm{SrAl}_{2} \mathrm{O}_{4}$ : $\mathrm{Eu}^{2+}, \mathrm{Dy}^{3+}$. J. Electrochem. Soc. 143, 2670 (1996).

22. Lyu, T. S. \& Dorenbos, P. Bi ${ }^{3+}$ acting both as an electron and as a hole trap in La-, Y-, and LuPO4. J. Mater. Chem. C. 6, 6240-6249 (2018).

23. Luo, H. D. \& Dorenbos, P. The dual role of $\mathrm{Cr}^{3+}$ in trapping holes and electrons in lanthanide co-doped $\mathrm{GdAlO}_{3}$ and $\mathrm{LaAlO}_{3}$. J. Mater. Chem. C. 6, 4977-4984 (2018).

24. Tanabe, S. \& Hanada, T. Appearance of light-storage rare earth aluminate phosphors and their optical properties. N. Ceram. 10, 27-33 (1996).

25. Takasaki, H., Tanabe, S. \& Hanada, T. Long-lasting afterglow characteristics of Eu, Dy codoped $\mathrm{SrO}_{-} \mathrm{Al}_{2} \mathrm{O}_{3}$ phosphor. J. Ceram. Soc. Jpn. 104, 322-326 (1996).

26. Wang, $X$. et al. $\mathrm{Gd}^{3+}$-activated narrowband ultraviolet-B persistent luminescence through persistent energy transfer. Dalton Trans. 50, 3499-3505 (2021).

27. Wang, F. et al. Simultaneous phase and size control of upconversion nanocrystals through lanthanide doping. Nature 463, 1061-1065 (2010).

28. Brites, C. D. S. et al. Instantaneous ballistic velocity of suspended Brownian nanocrystals measured by upconversion nanothermometry. Nat. Nanotechnol. 11, 851-856 (2016)

29. Ou, X. Y. et al. High-resolution $X$-ray luminescence extension imaging. Nature 590, 410-415 (2021).

30. Dvorak, M., Wei, S. H. \& Wu, Z. G. Origin of the variation of exciton binding energy in semiconductors. Phys. Rev. Lett. 110, 016402 (2013).

31. Chernikov, A. et al. Exciton binding energy and nonhydrogenic Rydberg series in monolayer WS2. Phys. Rev. Lett. 113, 076802 (2014).

32. Jiang, Z. Y. et al. Scaling universality between band gap and exciton binding energy of two-dimensional semiconductors. Phys. Rev. Lett. 118, 266401 (2017).

33. Robbins, D. J. \& Dean, P. J. The effects of core structure on radiative and nonradiative recombinations at metal ion substituents in semiconductors and phosphors. Adv. Phys. 27, 499-532 (1978).

34. Fidelis, I. \& Siekierski, S. The influence of enthalpy and entropy on the separation factor of the lanthanides in the $\mathrm{HEH} \varphi \mathrm{P}-\mathrm{HNO}_{3}$ system. J. Inorg. Nucl. Chem. 29, 2629-2635 (1967).

35. Dorenbos, P. Lanthanide charge transfer energies and related luminescence, charge carrier trapping, and redox phenomena. J. Alloy. Compd. 488, 568-573 (2009).

36. Thomas, D. G. \& Hopfield, J. J. Isoelectronic traps due to nitrogen in gallium phosphide. Phys. Rev. 150, 680-689 (1966).

37. Hopfield, J. J., Thomas, D. G. \& Lynch, R. T. Isoelectronic donors and acceptors. Phys. Rev. Lett. 17, 312-315 (1966).

38. Allen, W. J. Isoelectronic impurities in semiconductors: a survey of binding mechanisms. J. Phys. C: Solid State Phys. 4, 1936-1944 (1971).

39. Baldereschi, A. \& Hopfield, J. J. Binding to isoelectronic impurities in semiconductors. Phys. Rev. Lett. 28, 171-174 (1972). 